\title{
UNA MIRADA A LA SINCRONIZACIÓN DE LA RADIO COGNITIVA
}

\section{A LOOK AT THE SYNCHRONIZATION OF COGNITIVE RADIO}

\section{UM OLHAR SOBRE O TIMING DO RÁDIO COGNITIVO}

\author{
Danilo Alfonso López Sarmiento \\ dalopezs@udistrital.edu.co \\ Universidad Distrital Francisco José de Caldas \\ Bogotá D.C - Colombia
}

Leydy Johana Hernández Viveros

leydy.hernandez-v@uniminuto.edu.co

Corporación Universitaria Minuto de Dios- UNIMINUTO

Bogotá D.C - Colombia

Diagramación e ilustración de portada

Sindy Catherine Charcas Ibarra

Encuentre este artículo en:

http://revistas.uniminuto.edu/index.php/IYD

Para citar este artículo / To cite this article

López-Sarmiento, D; Hernández-Viveros, L. (2020). Una mirada a la sincroniza-

ción de la radio cognitiva. Inclusión \& Desarrollo, 7 (2), pp. 50-62
Fecha de recepción: 14 de marzo de 2020

Fecha de aceptación: 4 de junio de 2020

Fecha de publicación: 30 de junio de 2020 


\section{RESUMEN}

Objetivo. Proponer el uso de herramientas de inteligencia artificial como modelo para la sincronización de las etapas de decisión y compartición espectral de la radio cognitiva.

Metodología. La metodología que se utilizó fue exploratorio-descriptiva, recopilando información cronológicamente organizada en el tiempo y evaluando diferentes elementos que componen la radio cognitiva en cuanto a la sincronización entre sus etapas. De acuerdo a lo indagado y con el fin de llevar a cabo la investigación, se analizó cada una de las etapas que compone la radio cognitiva para una conexión y sincronización total, identificando en la etapa de decisión y compartición lo avanzado en el aspecto de integralidad entre las mismas.

Originalidad. De los estudios investigados basados en la radio cognitiva, no se explora en ningún caso la sincronización entre sus etapas para evidenciar una funcionalidad integral del sistema.

Limitaciones. Los diferentes estudios realizados a las etapas de la radio cognitiva deja ver que ninguno de estas por separado coindice, identificando que para el desarrollo de la propuesta se debe realizar el modelado para ambas etapas.

Resultados. Los resultados en la revisión literaria encontrados dan señas que esta sincronización no ha sido abordada dando espacio a un vacío de conocimiento que se pretende atacar.

Conclusión. La radio cognitiva es una metodología que propone el manejo dinámico del espectro radioeléctrico, integrando las etapas de detección, toma de decisiones, compartición y movilidad espectral. La fase de toma de decisiones espectrales se encarga de decidir cuál es el mejor canal disponible para transmitir los datos de los usuarios secundarios (SU) de manera oportunista, y su éxito depende de cuán eficiente sea el modelo de caracterización del usuario primario (PU) y la fase de compartición del espectro se encargará de esta manera de coordinar el acceso a el canal con otros usuarios, se busca la integralidad entre estas etapas.

Palabras clave: radio cognitiva, decisión espectral, compartición espectral, usuarios primarios, usuarios secundarios, inteligencia artificial, espectro radioeléctrico.

\section{ABSTRACT}

Objective. To propose the use of artificial intelligence tools as a model for the synchronization of the stages of decision and spectral sharing of cognitive radio.

Methodology. The methodology used was exploratory-descriptive, collecting information chronologically organized over time and evaluating different elements that make up the cognitive radio in terms of synchronization between its stages. According to what was investigated and in order to carry out the research, each of the stages that make up the cognitive radio was analyzed for a total connection and synchronization, identifying in the decision and sharing stage what is advanced in the aspect of comprehensiveness between them.

Originality. Of the investigated studies based on cognitive radio, the synchronization between its stages is not explored in any case to demonstrate an integral functionality of the system.

Limitations. The different studies carried out on the stages of cognitive radio show that none of these separately co-index, identifying that for the development of the proposal, modeling must be carried out for both stages.

Results. The results in the literary review found indicate that this synchronization has not been addressed giving space to a knowledge gap that is intended to attack.

Conclusion. Cognitive radio is a methodology that proposes the dynamic management of the radio spectrum, integrating the stages of detection, decision making, sharing and spectral mobility. The spectral 
decision-making phase is responsible for deciding which is the best available channel to opportunistically transmit secondary user (SU) data, and its success depends on how efficient the primary user (PU) characterization model is. and the spectrum sharing phase will be in charge of coordinating access to the channel with other users in this way, seeking comprehensiveness between these stages.

Key words: cognitive radio, spectral decision, spectral sharing, primary users, secondary users, artificial intelligence, radioelectric spectrum.

\section{SUMÁRIO}

Objetivo. Propor o uso de ferramentas de inteligência artificial como modelo para a sincronização das etapas de decisão e compartilhamento espectral do rádio cognitivo.

Metodologia. A metodologia utilizada foi de caráter exploratório-descritivo, coletando informações cronologicamente organizadas ao longo do tempo e avaliando diferentes elementos que compõem o rádio cognitivo em termos de sincronização entre suas etapas. De acordo com o que foi investigado e para realizar a pesquisa, cada uma das etapas que compõem o rádio cognitivo foi analisada para uma conexão e sincronização total, identificando na etapa de decisão e compartilhamento o que há de avançado no aspecto da abrangência. entre eles.

Originalidade. Dos estudos investigados com base no rádio cognitivo, a sincronização entre seus estágios não é explorada em nenhum caso para demonstrar uma funcionalidade integral do sistema.

Limitações. Os diferentes estudos realizados nas etapas do rádio cognitivo mostram que nenhum desses co-índices separadamente, identificando que, para o desenvolvimento da proposta, a modelagem deve ser realizada nas duas etapas.

Resultados. Os resultados da revisão literária encontrada mostram que essa sincronização não foi resolvida, dando espaço a uma lacuna de conhecimento que se pretende que seja atacada.

Conclusão. $O$ rádio cognitivo é uma metodologia que propõe o gerenciamento dinâmico do espectro de rádio, integrando as etapas de detecção, tomada de decisão, compartilhamento e mobilidade espectral. A fase de tomada de decisão espectral é responsável por decidir qual é o melhor canal disponível para transmitir oportunisticamente os dados do usuário secundário (SU), e seu sucesso depende da eficiência do modelo de caracterização do usuário primário (PU). e a fase de compartilhamento do espectro será responsável por coordenar o acesso ao canal com outros usuários dessa maneira, buscando a abrangência entre esses estágios.

Palavras chave: rádio cognitivo, decisão espectral, compartilhamento espectral, usuários primários, usuários secundários, inteligência artificial, espectro de rádio. 


\section{Introducción}

Al igual que la tierra se hace más costosa y escasa en áreas urbanas debido a que son densamente pobladas en razón a la calidad de vida ofrecida en esos sitios, el rango de operación del espectro radioeléctrico es más útil en ciertas bandas de frecuencia que en otras para las redes inalámbricas porque facilitan la interconexión de dispositivos disminuyendo la probabilidad de errores.

En la actualidad los sistemas inalámbricos se han caracterizado por una política de asignación espectral fija y regulada por el Gobierno de cada País; la cual presenta varias problemáticas relacionadas con el uso del espectro dentro de las que se encuentran: 1) Una cantidad importante de espectro no utilizado, como se ve en la Figura 1 donde se muestran las cifras típicas de ocupación espectral en la banda de los $30 \mathrm{MHz}$ a los 3 $\mathrm{GHz}$; 2) La utilización del espectro se concentra principalmente en las porciones que van desde los 88 a $216 \mathrm{MHz}$ y desde los 470 a $902 \mathrm{MHz}$ (Shared Spectrum Company, 2018), problema que se acentúa aún más a partir de las grandes variaciones espaciales y temporales en la ocupación espectral (Federal Communications Commission, 2003). La consecuencia de la subutilización del espectro es que hoy en día existe escases de este recurso ocasionando una degradación importante en la calidad del servicio ofrecido por las empresas de telecomunicaciones (ejemplo: banda celular); aspecto que ha motivado a investigadores de diferentes ramas a plantear posibles soluciones que permitan optimizar su aprovechamiento. Aparece como solución el acceso dinámico al espectro (DSA) y con ella el concepto de Radio Cognitiva (CR) donde su objetivo principal es identificar agujeros espectrales no utilizados por usuarios licenciados (PUs) para que puedan ser aprovechados de manera oportunista por usuarios no licenciados (SUs).

LaCR se puede definir como unsistema controlado por un proceso cognitivo capaz de percibir y procesar las condiciones existentes en el medio ambiente, para posteriormente ser utilizado por una técnica de aprendizaje capaz de optimizar el rendimiento de la red. Realizar esta tarea implica el uso de algoritmos altamente inteligentes capaces de tomar decisiones bajo diferentes condiciones en diferentes entornos de radio, además de otra serie de desafíos que necesitan ser resueltos (Fortuna \& Mohorcic, 2009).

Adicionalmente, la gestión espectral dinámica en CR incluye cuatro etapas principales (Khalid \& Anpalagan, 2010), donde la decisión espectral (que se encarga de seleccionar el mejor canal disponible a partir de los requisitos de calidad de servicio solicitados por el SU y reconfigurar el radio) tiene una importancia relevante debido a que es una de las fases que menos se ha investigado (López, Trujillo, \& Gualdron, Elementos fundamentales que componen la radio cognitiva y asignación de bandas espectrales., 2015), y que va a depender fundamentalmente de la caracterización del canal y del comportamiento estadístico de uso del canal por parte del PU.

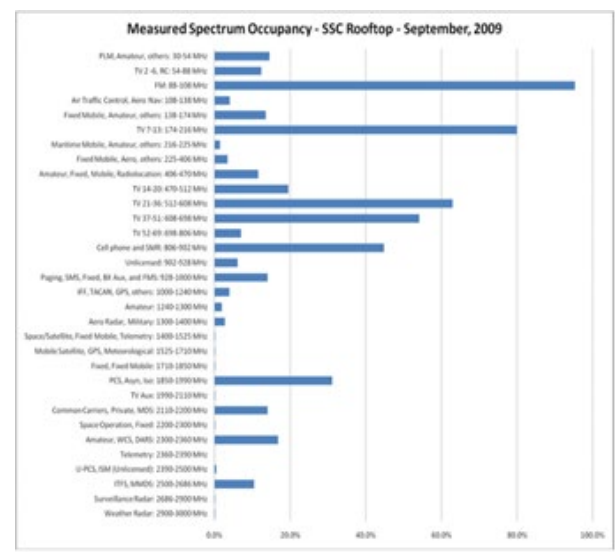

Fig. 1: Ocupación del espectro en los rangos $30 \mathrm{MHz}$ to $3 \mathrm{GHz}$ (Shared Spectrum Company, 2018).

Bajo lo anterior, cabe resaltar que existe literatura importante sobre el mejoramiento de las fases de la Radio Cognitiva de manera individual, pero existe un campo con baja exploración en la cual se aborda la integración de las fases, resaltando de esta manera la propuesta de la investigación la cual propone el uso de herramientas de inteligencia artificial para este fin. 


\section{Estado del arte}

\section{- Fases de la radio cognitiva}

En (Mitola, 1999), Mitola argumenta que una red basada en CR debe estar en la capacidad de usar el espectro de manera oportunista, valiéndose de su inteligencia y autonomía. Un radio cognitivo por definición debe estar compuesto por las etapas o fases mostradas en la figura 2:

- $\quad$ Sensado de espectro.

- Decisión de espectro.

- $\quad$ Compartición espectral.

- Movilidad del espectro.

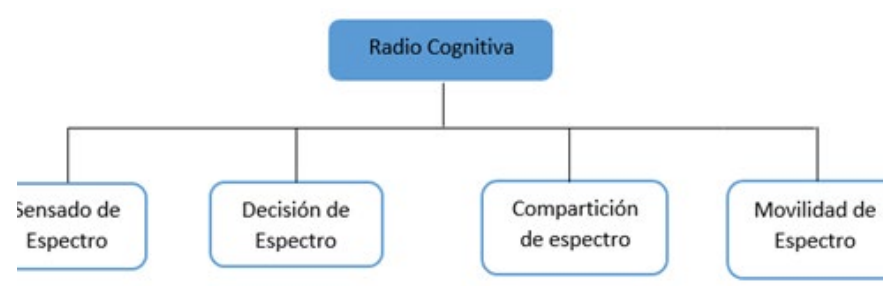

Fig. 2: Fases de un radio cognitivo (Fuente: Elaboración Propia)

Es importante recordar en este punto, que el objetivo principal de la radio cognitiva CR es compartir y aprovechar el espectro radioeléctrico. En esta estructura jerárquica, la propuesta se ubica en la fase de decisión y compartición espectral, las cuales se presentan en las figuras 3 y 4 identificando las tareas que cada uno de estas etapas debe realizar.

\section{- Decisión de Espectro}

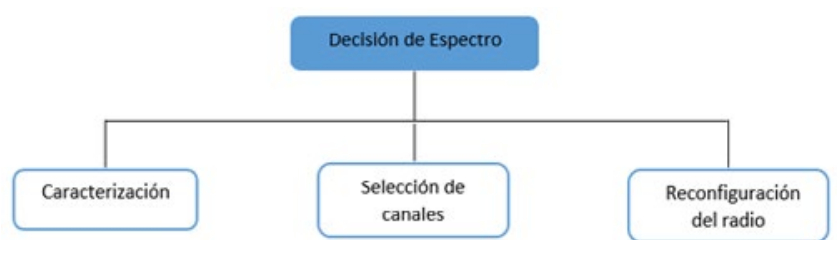

Fig. 3: Tareas de la fase de decisión espectral (Fuente: Elaboración Propia) Cabe mencionar que la función principal de la etapa de decisión es seleccionar el mejor canal disponible, comparando las características de cada una de las bandas con las requeridas por el usuario y valora cual es la mejor opción.

\section{- Compartición del Espectro}

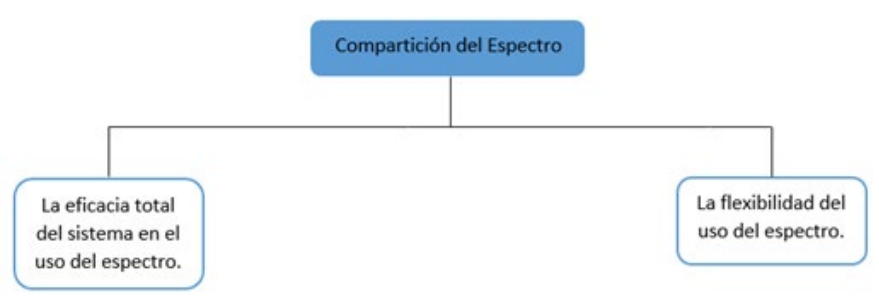

Fig. 4: Tareas de la fase de compartición del espectro (Fuente: Elaboración Propia)

Esta etapa o fase de la radio cognitiva, consiste en encontrar un método esquemático de distribución del espectro que sea equitativo y justo para todos los usuarios de la Radio Cognitiva sin interferir en las transmisiones de los usuarios con licencia. Éste es uno de los mayores retos a la hora de diseñar Radios Cognitivas.

Al abordar los conceptos y las tareas de las etapas, en especial la de decisión espectral, se debe definir con claridad el tipo de topología sobre el que se enfocará el estudio; en este sentido su clasificación depende de la forma en que se conectan entre sí los dispositivos: distribuida (Ad-Hoc) o centralizada (o basada en infraestructura). En una estructura AdHoc, los SUs se comunican directamente entre sí, sinlanecesidad derequerir de un dispositivo central que gestione; compartiendo su conocimiento local con aquellos dispositivos que se encuentren dentro del rango de alcance. A partir de su percepción de la red, cada radio puede decidirse por la selección de un canal de acuerdo a sus necesidades. Si la solicitud no es satisfecha a causa de la aparición de un PU o por degradación del canal, se tiene la posibilidad de repetir todo el proceso empezando de nuevo desde la monitorización del espectro (López, 2017).

En un sistema basado en infraestructura, todos los usuarios dentro de la zona de funcionamiento, 
gestionan sus solicitudes de decisión a través de un dispositivo central, como sucede en la especificación IEEE 802.22 (Red Inalámbrica de Área Regional-WRAN). Como la red de radio cognitiva (CRN) opera dentro de la región de cobertura de los usuarios licenciados, utiliza técnicas de DSA para acceder oportunamente al espectro de red principal sin causar interferencia perjudicial a los licenciados al igual que en el caso Ad-Hoc. Para ello, los SUs realizan observación del espectro en los en las bandas especificadas, para luego enviarlas a la estación base (BS) que actúa como un centro de difusión (Caur, Buttar, $\&$ Anand, Spectrum sharing schemes in cognitive radio network: A survey., 2018). Tanto la BS como sus clientes asociados pueden detectar la presencia de las PUs usando técnicas de detección como el basado en energía, ciclo estacionalidades, o bases de datos de geo-localización, entre otros. En algunos casos como en (Pedraza, 2016), se utilizan dos medios físicos, uno para observar el comportamiento del canal primario y el otro para actualizar constantementelaBS. Unavezanalizada la información y se tenga claridad de los canales disponibles, la BS construirá la lista definitiva de las bandas junto con la calidad de servicio (QoS) disponible en cada caso, con el fin de asignarlos a cada SU a partir de sus requerimientos.

\section{Marco teórico}

En las últimas dos décadas se ha venido observando un crecimiento cada vez mayor (y con un comportamiento exponencial creciente (figura 5) en el número de personas, empresas, industrias que dependen de la súper-autopista de las telecomunicaciones para interactuar con el mundo que los rodea. Esta tendencia global, junto con un crecimiento continuo en la demanda de servicios de voz, video y datos móviles, de diversas formas ha venido imponiendo nuevos desafíos a los ingenieros que se desempeñan en el área (López, Anzola, Zapata, \& Rivas, 2018). Tal es el impacto de la Internet que se ha convertido en el componente más importante que engloba la economía mundial; esto implica que satisfacer la demanda de los usuarios manteniendo un buen nivel de calidad del servicio es uno de los retos que los investigadores continuamente enfrentan.

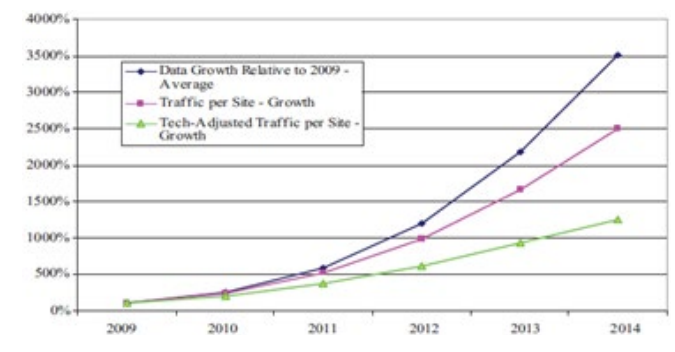

Fig. 5: Crecimiento exponencial del tráfico (Federal Communications Commission, 2003)

Toda esta tendencia, ha demarcado una clara dependencia del ser humano por el acceso a la información, preferiblemente desde dispositivos móviles (figura 6); variable que ha generado como consecuencia la demanda de más y mejores servicios inalámbricos impactando de manera negativa el canal debido a la falta de espectro útil para el transporte de la data, deteriorando el rendimiento.

En este sentido, tradicionalmente, el espectro de radio frecuencia (RF) es administrado por las agencias gubernamentales mediante la asignación de partes fijas del espectro a usuarios individuales en forma de licencias renovables, convirtiendo casi en un imposible la asignación de nuevas bandas que le permitan a las redes inalámbricas su normal desarrollo, y de conseguirse, la competencia por estebienhahechoquesucostosea elevado.Aunque dicho enfoque regulador asegura comunicaciones libres de interferencia entre terminales de radio, adolece por la utilización ineficiente del espectro.

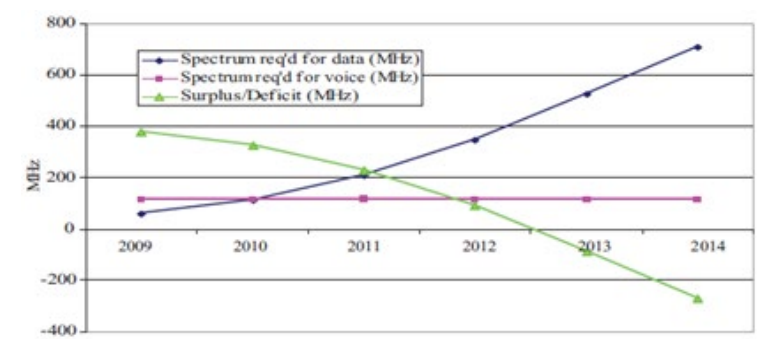

Fig. 6: Estimación de la utilización del espectro de acuerdo con la FCC (Federal Communications Commission, 2003) 
La comisión federal de Comunicaciones de los Estados Unidos (FCC), argumenta que, aunque la capacidad espectral parece estar acabando, muchas de las frecuencias existentes en las bandas con licencia son poco utilizadas (Federal Communications Commission, 2003). Estos supuestos son corroborados por los estudios realizados en (Cao, Lu, Wen, Lei, \& Hu, 2018), (Arulkumaran, Deisenroth, Brundage, \& Bharath, 2017), donde se certifica que la realidad de la escasez, es más bien una apreciación burocrática, a pesar de que este recurso natural es finito, y que podría mejorarse su uso permitiendo la coexistencia entre clientes licenciados y no licenciados. Esta interacción lleva consigo un cambio de paradigma de asignación estática del espectro a una distribución dinámica; tarea que trae enormes retos, ya que implica cumplir con la exigencia de no interferencia para los clientes que pagan por él.

Para tal fin, el paradigma de radio cognitiva (CR) (Mohammadi, AlFuqaha, \& Guizani, 2018), se ha propuesto como estrategia al establecimiento e implementación de soluciones técnicas, tendientes a beneficiar la eficiencia espectral en sistemas inalámbricos presentes y futuros de manera dinámica.

\section{- Radio Cognitiva}

Desde mediados de los años 90, la radio cognitiva (CR) ha recibido considerable atención por parte de la comunidad científica como la técnica que permite la gestión eficiente del espectro de radiofrecuencia y su éxito dependerá de que tan eficiente y ágil sea el proceso de acceso dinámico al espectro (Maglogiannis, Naudts, Shahid, \& Moerman, 2018). Estructuralmente la CR esta soportada en la radio definida por software (SDR) pero con capacidades para aprender de su entorno operativo y adaptarse a las variaciones estadísticas de acuerdo con los estímulos de entrada en busca de la utilización eficaz de los componentes de la red (Cao, Lu, Wen, Lei, \& Hu, 2018). Tecnológicamente la CR aparece como posible solución a la implementación real del concepto de acceso dinámico al espectro (DSA); lo que da evidencia de la existencia de una clara diferencia entre estos dos últimos conceptos, donde el DSA se relaciona con estándares o metodologías que proponen cambiar la forma de gestionar el espectro a un modo más eficiente; por el contrario la CR es considerada como la tecnología capaz de llevar a la realidad el paradigma planteado por DSA; y para ello se han establecido de manera general dos diferentes políticas de transmisión (subyacente y de superposición) que pretenden garantizar la adecuada implementación de la CR, minimizando el riesgo de posible interferencia o colisión generada desde los usuario secundarios (SU) y hacia los usuarios primario (PU), donde la finalidad común para los dos casos es el uso del acceso oportunista sin interferir las transmisiones de los PUs (Gao, Wen, Huang, Chen, \& Su, 2017).

La radio cognitiva haciendo uso de espectro subyacente, es una técnica de gestión en la que los SUs usan señales de muy baja densidad espectral con el fin de coexistir en la misma banda con los PUs (que tienen la propiedad de utilizar densidades de mayor potencia) lo que implica, que el envío simultaneo de información se lleva a cabo si la interferencia generada desde el SU no sobrepasa un umbral preestablecido. En este caso la consecuencia de que convergen usuarios heterogéneos en el mismo instante de tiempo se relaciona con el incremento en el ruido de fondo para el PU (Khomh, Adams, Cheng, \& Fokaefs, 2018).

Laradiocognitivamediantesuperposiciónespectral, se refiere a la posibilidad de uso del espectro licenciado o privado, solo en aquellos instantes en los que el PU no esté haciendo uso del mismo. Para ello es clave que el SU tenga conocimiento previo de la dinámica del comportamiento del tráfico del PU, a través de la caracterización o estimación de uso del canal. La diferencia entre uno y otro método para la implementación de la CR, tiene que ver más con el tipo de información que requieren los SUs para evitar interferencias con el PU y de esta manera poder aprovechar al máximo el canal subutilizado. Para las redes basadas en el método 
subyacente, se imponen estrictos umbrales máximos para la potencia que puede ser usada por los SUs disminuyendo la posibilidad de interferencias; para las redes que usan un control mediante superposición, los nodos cognitivos disminuyen los niveles de ruido o interferencia usando técnicas de codificación.

Por tanto, una de las funciones destacadas de un equipo con soporte para CR, es la de encontrar oportunidades espectrales (figura 7) para poder acceder a ellos, sin interferir con el usuario dueño de la banda. Un agujero de espectro se define como un rango de frecuencia concedida a un PU, pero que en un momento determinado y ubicación geográfica específica no está siendo utilizado (Cao, Lu, Wen, Lei, \& Hu, 2018).

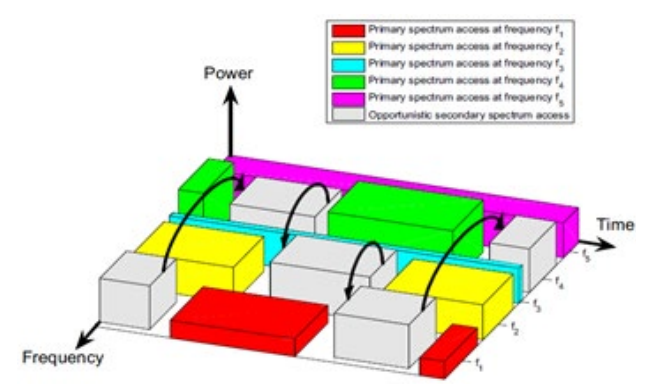

Fig. 7: Concepto de acceso oportunista al espectro (Peysakhovich \& Naecker, 2017)

En (Mitola, 1999), argumenta que una red basada en CR se define como una estructura compleja en la que los dispositivos son capaces de adaptarse al entorno. Dentro de las características de adaptabilidad, está la capacidad de usar el espectro de manera oportunista, valiéndose de su inteligencia y autonomía. En general, un sistema de radio cognitivo debe ser capaz de realizar cuatro tareas de manera eficiente: sensado, decisión, compartición y movilidad de espectro.

La detección del espectro o sensado de espectro, determina la porción del espectro disponible. Este hace un barrido de las frecuencias en el área de interés para identificar los "white spaces" con mayor probabilidad de ser utilizados en un determinado espacio de tiempo, frecuencia $y$ potencia dentro de una región geográfica específica (Gopakumar, Tiwari, \& Rahman, 2018).

La decisión de espectro, selecciona el mejor canal disponible. Este se relaciona con la selección del canal o grupo de canales de acuerdo a dos factores: 1) las características disponibles en el entorno; 2) Las necesidades solicitadas por el SU para el transporte de los datos.

La compartición del espectro, coordina el acceso al canal escogido con otro usuario. Esta consiste en asignar de manera adecuada y equitativa las bandas de frecuencia maximizando su uso sin generar interferencia en los PUs ni entre usuarios cognitivos (Gopakumar, Tiwari, \& Rahman, 2018).

La movilidad de espectro, es la capacidad del CR para dejar una porción del espectro utilizado cuando un PU lo requiere, y además buscar otro espacio vacío adecuado para continuar con la comunicación.

\section{- Avances en el desarrollo de la radio cognitiva utilizando metodologías de inteligencia artificial}

En concordancia con las definiciones anteriores, el proceso de lograr una utilización eficiente del espectro con CR requiere un marco de gestión del espectro dinámico (DSMF) (Caur, Buttar, \& Anand, 2018) que no solo incluye el funcionamiento individual e independiente de cada etapa, sino la interacción y sincronización entre las partes (figura 8) para que el sistema espectral funcione de manera integral.

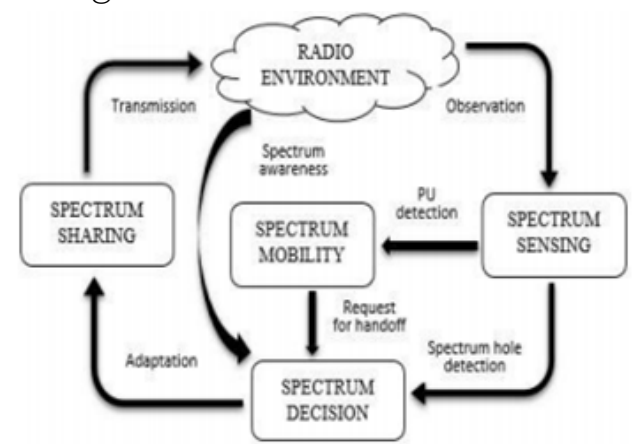

Fig. 8: Marco de gestión de espectro dinámico en CR (Caur, Buttar, \& Anand, 2018) 
Desde el punto de vista individual, para cada una de las etapas que compone la CR existen diversas propuestas que plantean soluciones para realizar un adecuado sensado de espectro, una toma de decisiones acorde con las necesidades (López, 2017), una asignación y compartición de canales sujeto a la disponibilidad de recursos y un handoff espectral que puede ser de tipo reactivo o proactivo. No obstante, hoy en día la inteligencia artificial (IA) (Cao, Lu, Wen, Lei, \& Hu, 2018) está revolucionando la forma de abordar y resolver problemas u optimizar procesos que requieran un razonamiento de nivel superior como es el caso de CR aplicando técnicas como Deep Reinforcement Learning, Q-Learning, Machine Learning, y otras técnicas como (Gopakumar, Tiwari, \& Rahman, 2018); temas que muy poco se han abordados dentro de las CRNs.

\section{- $\quad$ Avances en la sincronización del sistema}

En (Stergios \& Arumugam, 2011), se centran en la maximización del rendimiento del espectro que comparte redes de radio cognitivas y proponen un nuevo sistema de radio cognitivo que mejora significativamente su rendimiento alcanzable. Presentan un receptor y una estructura de trama novedosos para redes de radio cognitivas de intercambio de espectro y estudian el problema de derivar la estrategia de asignación de potencia óptima que maximiza la capacidad ergódica del sistema de radio cognitivo propuesto bajo las restricciones de potencia de interferencia y transmisión promedio. También observan la capacidad de interrupción del sistema de radio cognitivo propuesto bajo varias restricciones que incluyen las limitaciones de potencia de interferencia y transmisión promedio, y las restricciones de potencia de interferencia máxima.

En (Mohd \& Mohd, 2012), proporciona un promedio de lafunción de distribución acumulativa inversa máximo-mínimo (ICDF). Usan coseno elevado para probar el rendimiento del detector de señal para realizar la simulación. Se encontró que el detector de señales SVD basado en valores propios, era más eficiente en la detección de señales sin conocer las propiedades de la señal transmitida.

En 2012, Shaw et al, proponen DCR-Sync, un nuevo protocolo de sincronización de tiempo para CRN. A diferencia de las propuestas existentes, DCRSync está totalmente distribuido y es resistente a la falla de los nodos raíz, es decir, los nodos que desempeñan el papel de maestro en el proceso de sincronización. Presentan DCR-Sync en dos versiones. La primera versión es de naturaleza estática, y la segunda versión puede adaptarse dinámicamente a los cambios de red. A través de simulaciones extensas, mostramos que ambas versiones superan el rendimiento de los protocolos de sincronización existentes. Precisamente, ambas versiones de DCR-Sync se simulan utilizando el simulador NS2 y se comparan con el protocolo TPSN. Los resultados de la simulación muestran las mejoras obtenidas por DCR-Sync en términos de sobrecarga de red y tiempo de convergencia.

En 2014, Chin et al. presenta un esquema de detección de señal OFDM asistida por sincronización iterativa para aplicaciones de radio cognitiva $(C R)$ en canales de múltiples rutas en regiones de baja SNR. Para detectar la señal OFDM, se emplea una prueba de relación de probabilidad logarítmica (LLR) sin símbolos piloto adicionales utilizando el prefijo cíclico (CP). Los resultados analíticos indican que el LLR de las muestras recibidas a una SNR baja se puede aproximar por sus funciones de loglikelihood (LL), lo que nos permite estimar los parámetros de sincronización para la detección de señales. La función LL es compleja y depende de varios parámetros, incluido el coeficiente de correlación, el desplazamiento de frecuencia de portadora (CFO), el desplazamiento de temporización de símbolo y la longitud del canal. La descomposición de un problema de sincronización en varios subproblemas de estimación de parámetros relativamente simples elimina una búsqueda de cuadrícula multidimensional. También se diseña un esquema iterativo para implementar el proceso 
de sincronización. Los resultados de la simulación confirman la efectividad del detector propuesto.

\section{Metodología}

El presente artículo plantea la aplicación de diferentes enfoques metodológicos. El primero de ellos es decarácter exploratorioy descriptivoconel fin de realizar una selección de las propuestas más destacadas existentes mediante la generación de un estado del arte; el segundo es de tipo analítico que tiene por objetivo el estudio detallado de los modelos/técnicas/algoritmos más relevantes y con mejor respuesta en cada una de las etapas que involucra la CR; el tercero es de carácter investigativo con el que se pretende la selección de técnicas basadas en inteligencia artificial para la implementación de las etapas de decisión y compartición espectral, que conformaran el sistema de radio cognitivo buscando con ello mejorar el rendimiento del sistema; el cuarto es de tipo experimental a través de la integración y evaluación del framework o modelo de gestión espectral.

Para (Hernández Sampieri, Collado, \& Lucio, 2010) "los métodos mixtos representan un conjunto de procesos sistemáticos, empíricos y críticos de investigación e implican la recolección y el análisis de datos cuantitativos y cualitativos, así como su integración y discusión conjunta, para realizar inferencias producto de toda la información recabada (metainferencias) y lograr un mayor entendimiento del fenómeno bajo estudio"

\section{Resultados}

Cabe destacar que en la revisión literaria consultada no se encontraron indicios que resolvieran el problema de la selección proactiva de canales a partir de la probabilidad de arribo de usuarios secundarios a la estación base (BS), lo que implícitamente requiere la sincronización entre estas variables, junto con las etapas de decisión y compartición espectral para que el sistema funcione de una manera eficiente.

Lo proyectado se centra en la parte de decisión espectral, específicamente como lo muestra la figura 9, en la:

Caracterización de usuarios secundarios, con el fin de generar un modelo (a partir del uso histórico del canal), para la asignación equitativa de la banda espectral por parte de los usuarios cognitivos dentro de la CRN.

Selección del canal proactivo, utilizando un modelo predictor donde el algoritmo sea capaz anticipadamente de elegir la banda adecuada (dependiendo del tráfico del SU) a ser asignada en un futuro a ese usuario cognitivo. Esto permitirá disminuir el tiempo que se tarda en colocar los datos en el medio inalámbrico para su transferencia.

Yenlaparte decompartición delespectroutilizando metodologías de IA, se encuentre la sincronización, elevando el rendimiento y desempeño de las CRNs, debido a que, reducen el tiempo de asignación de canales, además de disminuir la cantidad de colisiones entre SUs y PUs.

La explicación del diagrama a bloques de la figura 9 es como sigue.

La topología de trabajo será centralizada.

Existen oportunidades espectrales disponibles para soportar las transmisiones (sensado de espectro).

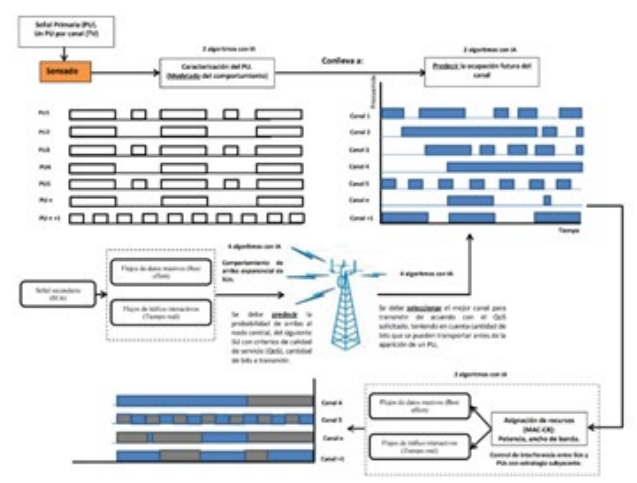

Fig. 9: Sincronización Esperada entre las etapas de decisión y compartición espectral (Fuente: Elaboración Propia)

\section{Conclusiones}

En relación con la revisión literaria estudiada y planteada desde lo esperado existe un aspecto no abordado en la existencia de algoritmos basados en inteligencia artificial que permitan 
la sincronización de las subetapas de selección proactiva de canales y estimación del arribo de SUs, además de la interrelación entre las fases de decisión y compartición espectral, para el transporte adecuado de los datos de usuarios.

A manera de conclusión y en concordancia con la literatura la CR es una de las técnicas más recientes que administra de manera más eficiente los recursos mediante la gestión dinámica del espectro. Un número importante de investigaciones se ha enfocado en proponer soluciones de manera individual a cada una de las problemáticas existentes en cada etapa que compone la CR, sin poder llegar a evaluar y validar el comportamiento de los modelos desarrollados dentro del funcionamiento de la CR como un "sistema integral", razón de peso para proponer un modelo que pueda garantizar la interacción entre las etapas de decisión y compartición espectral.

Si bien es cierto, siguen apareciendo múltiples propuestas (tanto cualitativas como cuantitativas) para optimizar de manera individual el funcionamiento de cada etapa de las redes de radio cognitiva (CRNs) con resultados interesantes, existe una carencia en la generación de investigaciones de modelos de gestión de espectro que permitan estudiar integralmente el proceso que involucra el diseño de un sistema de radio cognitiva en sus etapas de decisión y compartición espectral, aplicando metodologías basadas en IA, teniendo la posibilidad con ello de evaluar aspectos que no han sido adecuadamente abordados y que son de suma importancia al implementar el concepto de radio cognitiva a nivel real como lo es la sincronización.

Cabe destacar que lo proyectado busca lograr la integralidad entre dos de las cuatro etapas que componen la radio cognitiva, las cuales son: etapas de decisión y compartición espectral. 


\section{Referencias bibliográficas}

Arulkumaran, K., Deisenroth, M., Brundage, M., \& Bharath, A. (2017). Deep reinforcement learning: A brief survey, Vol. 34 , no. 6,. IEEE Signal Processing Magazine, Vol. 34 (Nº 6), 26-38.

Cao, G., Lu, Z., Wen, X., Lei, T., \& Hu, Z. (2018). AlF: An artificial intelligence framework for smart wireless network management. IEEE Communications Letters, Vol. 22( $\left.N^{\circ} 2\right), 400-403$.

Caur, R., Buttar, A., \& Anand, J. (2018). Spectrum sharing schemes in cognitive radio network: A survey. Proceeding of the 2 nd International Conference on Electronics Communications and Aerospace Technology, 1279-1284.

Caur, R., Buttar, A., \& Anand, J. (2018). Spectrum sharing schemes in cognitive radio network: A survey. Proceeding of the 2 nd International Conference on Electronics Communications and Aerospace Technology, 1279-1284.

Chin, W., Kao, C., Chen, H., \& Liao, T. (2014). Iterative SynchronizationAssisted Detection of OFDM Signals in Cognitive Radio Systems. Vehicular Technology, IEEE Transactions, 63(4), 1633-1644.

Federal Communications Commission. (2003). Notice of proposed rulemaking and order. Mexico D.F: Report ET Docket No: 03-332.

Fortuna, C., \& Mohorcic, M. (2009). Trends in the development of communication networks: Cognitive networks. Journal Computer Networks.,, 53(N 9), 1354-1376. doi: https://doi.org/10.1016/j.comnet.2009.01.002

Gao, Z., Wen, B., Huang, L., Chen, C., \& Su, Z. (2017). Q-Learning-based power control for LTE enterprise femtocell networks. IEEE Systems Journal, Vol. 11(No. 4), 2699-2707.

Gopakumar, V., Tiwari, S., \& Rahman, I. (2018). A deep learning based data driven soft sensor for bioprocesses. Journal Biochemical Engineering, 28-39.

Hernández Sampieri, R., Collado, C. F., \& Lucio, M. d. (2010). Metodología de la investigación. México D.F: McGraw Hill.

Khalid, L., \& Anpalagan, A. (2010). Emerging cognitive radio technology: Principles, challenges and opportunities. Elsevier, Computers and Electrical Engineering, 38(2), 358-366. doi: https://doi.org/10.1016/j. compeleceng.2009.03.004

Khomh, F., Adams, B., Cheng, J., \& Fokaefs, M. (2018). Software Engineering for Machine-learning applications: The road ahead. IEEE Software, Vol. 35(No. 5), 81-84.

López, D. (29 de Septiembre de 2017). Tesis doctoral: Implementación de un modelo predictor para la toma de decisiones en redes inalámbricas de radio cognitiva. Obtenido de Facultad de Ingenierías, Universidad Distrital Francisco José de Caldas: https://doctoradoingenieria.udistrital.edu.co/index.php/ es/inicio/documentos/repositorio-de-tesis-doctoral/item/488-implementacion-de-un-modelo-predictor-para-la-toma-de-decisiones-en-redes-inalambricas-de-radio-cognitiva López, D., Anzola, C., Zapata, D., \& Rivas, E. (2018). Designing a MAC algorithm for equitable spectrum allocation in cognitive radio wireless networks. Journal Wireless Personal Communications, Vol. 98( $\mathrm{N}^{\circ}$ 1), 135-145.

López, D., Trujillo, E., \& Gualdron, O. (2015). Elementos fundamentales que componen la radio cognitiva y asignación de bandas espectrales. Información tecnológica, 26(1), 23-40. doi: http://doi.org/10.4067/ S0718-07642015000100004

Maglogiannis, V., Naudts, D., Shahid, A., \& Moerman, A. (2018). Q-Learning scheme for fair coexistence between LTE and Wi-Fi in unlicensed spectrum. IEEE Access, Vol. 6, 27278-27293.

Mitola, J. (1999). Software radios - survey, critical evaluation and future directions. Proceedings of the National Telesystems Conference (NTC 1992, 13-23.

Mohammadi, M., AlFuqaha, A., \& Guizani, M. (2018). Semisupervised deep reinforcement learning in 
support of IoT and smart city servicesl, vol. 5 , no. 2,. IEEE Internet of Things Journal, Vol. 5(No. 2), 624635.

Mohd, F., \& Mohd, S. (2012). SVD Detection for Cognitive Radio Network based on Average of MaximumMinimum of the ICDF. International Journal of Advanced Computer Research (IJACR), 5.

Pedraza, L. (16 de 08 de 2016). Tesis doctoral: Modelo de propagación para un entorno urbano que identifica las oportunidades espectrales para redes móviles de radio cognitiva. Obtenido de Facultad de Ingeniería, Universidad Nacional de Colombia: http://bdigital.unal.edu.co/view/subjects/34.html Peysakhovich, A., \& Naecker, J. (2017). Using methods from machine learning to evaluate behavioral models of choice under risk and ambiguity. Journal of Economic Behavior and Organization, 373-384.

Shared Spectrum Company. (2018). Spectrum reports: Spectrum occupancy measurement. General survey of radio frequency bands (30 MHz to $3 \mathrm{GHz}$ ). Vienna, Virginia: Shared Spectrum Company. Obtenido de http://www.sharedspectrum.com/wp-content/uploads/2010_0923-General-Band-Survey-30MHzto-3GHz.pdf

Shaw, S., Ghamri-Doudane, Y., Santos, A., \& Nogueira, M. (2012). A reliable and distributed time synchronization for Cognitive Radio Networks. Global Information Infrastructure and Networking Symposium (GIIS), 1-19.

Stergios, S., \& Arumugam, N. (2011). Enhancing the Capacity of Spectrum Sharing Cognitive Radio Networks. IEEE Transactions on Vehicular Technology, 1-10. 